\title{
Preliminary Results From The More Advanced Control Advice Project For Secure Operation Of Isolated Power Systems With Increased Renewable Energy Penetration And Storage
}

\author{
N. Hatziargyriou, G. Contaxis, M. Matos, J.A. Peças Lopes, M. H. Vasconcelos, G. Kariniotakis, D. \\ Mayer, J. Halliday, G. Dutton, P. Dokopoulos, A. Bakirtzis, J. Stefanakis, A. Gigantidou, \\ PhilipO’Donnell, Damian McCoy, M.J. Fernandes, J.M.S. Cotrim, A.P.Figueira
}

\begin{abstract}
In this paper, preliminary results from MORE CARE, a European R\&D project financed within the Energy Program are described. This project has as main objective the development of an advanced control software system, aiming to optimize the overall performance of isolated and weakly interconnected systems in liberalized market environments by increasing the share of wind energy and other renewable forms, including advanced on-line security functions. The main features of the control system comprise advanced software modules for load and wind power forecasting, unit commitment and economic dispatch of the conventional and renewable units and on-line security assessment capabilities integrated in a friendly Man-Machine environment. Pilot installations of advanced control functions are foreseen on the islands of Crete, Ireland and Madeira.
\end{abstract}

Index Terms - Utility-Integration, Control Systems, Forecasting Methods, Operational Planning, Security Assessment.

\section{INTRODUCTION}

The population of EU islands is about 12 million persons without taking into account the British islands and Ireland. These islands are characterized by high costs of electricity production because they are based on imported fuel, mainly diesel. The import costs are further increased by the cost of transportation. On the other hand, many islands possess a significant potential of local resources of wind and solar energy, which, if used to produce electricity, contributes to their economic development and moreover helps protecting their fragile environment. However, there is a number of technical problems limiting this strategy: the grid is relatively weak, wind is a volatile power source, reserve needs to be a high percentage of the installed power and security might face increased risks. In consequence, these systems tend to be managed in a conservative way that underexploits the renewable energy sources and increases the already large costs of electricity in islands. Alternatively, the system might be operated with lower security margins. Advanced control systems can substantially help operators to manage efficiently these systems allowing increased pene-

N. Hatziargyriou and G. Contaxis are with the National Technical University of Athens, Greece. M Matos, J. A. Peças Lopes are with the Faculty of Engineering of Porto University and the with the Power Systems Unit of INESC Porto, Portugal. Helena Vasconcelos is also with INESC Porto. G. Kariniotakis and D. Mayer are with Armines in France. G. Halliday and G. Dutton are with the CCLRC-RAL, UK. P. Dokopoulos, A. Bakirtzis are with the AUTH in Greece. J. Stefanakis and A. Gigantidou are with PPC - Greece. PhilipO'Donnell and Damian McCoy are with ESB in Ireland. M.J. Fernandes, J.M.S. Cotrim and A.P.Figueira are with EEM in Portugal. tration of renewable energy sources in a secure way, as shown initially in [1]. In particular, the advanced control system "CARE" has been developed previously and installed in Crete, an island with a peak load exceeding 400 MW and more than $60 \mathrm{MW}$ of installed Wind Power [2,3].

The objectives of MORE CARE, financed within the Energy Program, (Contract ERK5-CT1999-00019) are to produce enhanced capabilities of the CARE software. This aims at optimizing the overall performance of isolated and weakly interconnected systems in liberalized market environments by increasing the share of wind energy and other renewable forms, taking into account pumped hydro storage facilities and providing advanced on-line security functions, both in preventive and corrective mode.

More specifically, the work comprises collection and analysis of renewable, electrical and operating data and identification of the needs for the following developments of on-line control functions:

- Improved wind power forecasting modules for shortterm (0-8 h) and medium time (4-48 h) horizons.

- Hydro power forecasting functions.

- Unit Commitment and Economic Dispatch modules that take into account the availability of hydrostorage, liberalized market conditions and increased security conditions.

- On-line security modules that provide both preventive and remedial advice in case of predetermined disturbances.

- Installation of the enhanced and new forecasting, operational planning and security modules on Crete, in order to face the new operating conditions.

- Installation of the enhanced and new forecasting, operational planning and security modules on Madeira, in order to face effectively the operating conditions with very high wind power penetration.

- Development of wind power forecasting modules for the power system of Ireland.

In this paper a general description of the software, including functionalities, general constraints, the characteristics of the user, operational environment, etc. are provided.

\section{ThE CARE SYSTEM ARCHITECTURE}

The MORE CARE system aims to assist the operators of island systems by proposing optimal operating scenarios for the various power units, as well as the various actions needed to avoid dangerous situations, which might result 
from a poor prediction of load or weather or pre-selected disturbances. The insurance of increased security and reliability of the system will allow maximization of renewable penetration. The product under development includes various modules of forecasting, operational planning and security assessment. Due to the diverse needs of targeted medium and large scale systems, the software under development is highly modular, allowing integration of the options that are best suited to the particularities of each system.

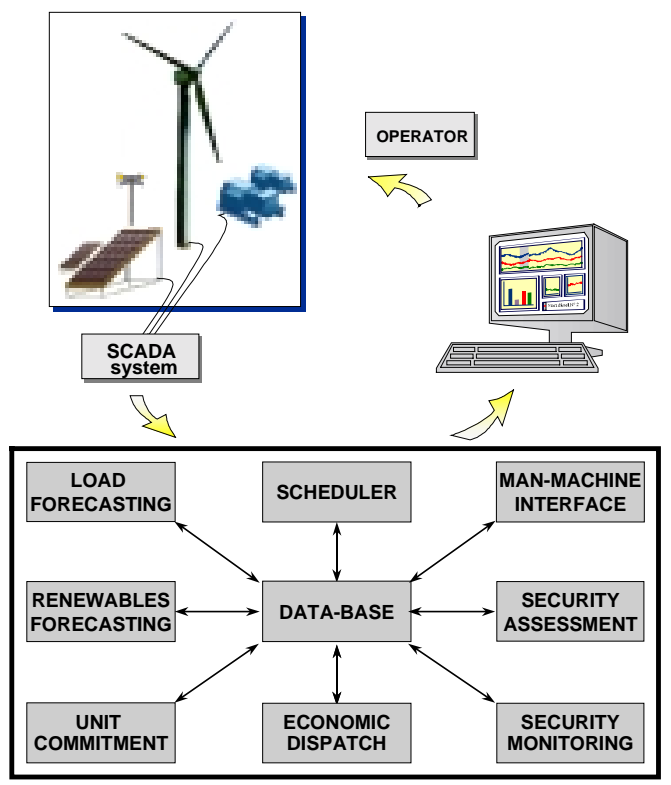

Fig. 1: The CARE system architecture.

Fig. 1 shows the general CARE system architecture, also retained in the MORE CARE system and the various functions that will be implemented. Figure 2 shows the execution cycles and the succession of the MORE CARE modules to generate the power system operation schedules. This flowchart is appropriate for relatively larger systems comprising steam and diesel or gas units. The power system of Crete is typical of such island systems. Units requiring both longer and shorter scheduling times characterize these systems, therefore both longer and shorter horizon forecasts and unit commitment functions are included. For island systems comprising only diesel units or gas turbines, e.g. the Madeira system, it is possible to simplify these execution cycles.

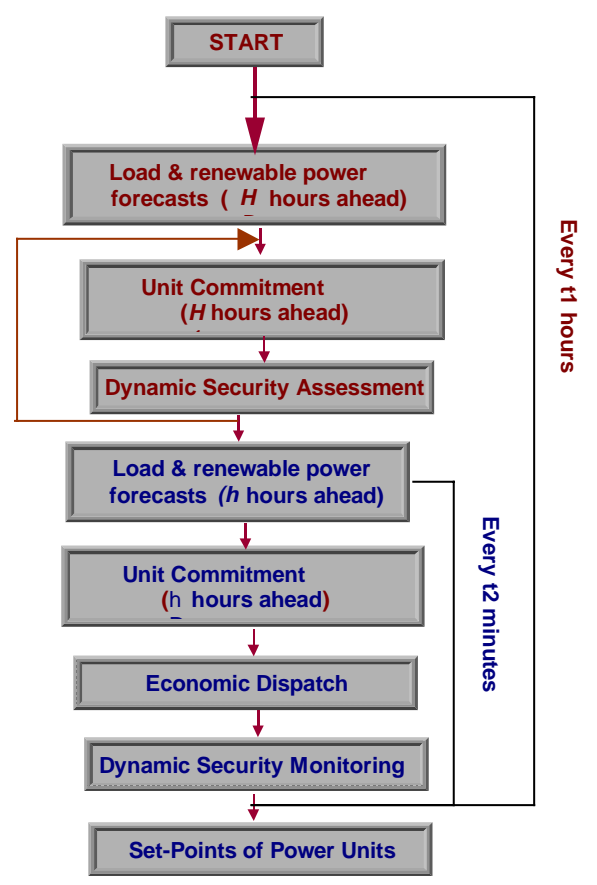

Fig. 2 : Main operations of the MORE CARE system algorithm. (For the pilot control installation of Crete it will be $: H=48$ hours, $h=4$ hours, $t 1=1$ hour, $\mathrm{t} 2=20$ minutes.)

Unit commitment will have an horizon of 8 hours ahead (moving window) but tests showed that an outer cycle of 48 hours was needed to define guidelines that take into account the daily cycle of the load.

Security assessment follows the unit commitment and dispatch modules, leaving to the operator the decision whether or not he wants to activate the module for validation of the proposed dispatch (or pre-dispatch resulting from the unit commitment). In this case, another solution will be presented to the operator if the first one is considered insecure.

In the next sections, the main modules of the system are described.

\section{LOAD FORECASTING}

In order to perform the Unit commitment and Economic Dispatch functions for the next planning horizon, it is necessary to have a forecast of the profile of the total load of the power system for this horizon. As "forecasted load profile" is characterized a set of consecutive spot forecast values as well as confidence intervals for these forecasts.

The following three general load forecasting requirements are defined for the load forecasting (LF) module:

1. One forecast for the next time step (20 minutes) to be used by the Economic Dispatch (ED) module.

2. Forecasts for the short-term horizon (4 hours) with the basic time-step (20 minutes) to be used by the short-term Unit Commitment function (ST-UC).

3. Forecasts for the long-term horizon (48 hours) with hourly time-step to be used by the long-term Unit Commitment function (LT-UC).

The above forecasts are generated with a sliding window scheme at time intervals equal to the basic time-step (every 20 minutes). The sliding window scheme is a particular feature of the MORE CARE forecasting module compared to forecasting functions for conventional systems. Usually in conventional systems forecasts are produced once per day. 
In autonomous systems with high penetration, it is necessary to update forecasts several times per day.

The LF module also integrates a pre-processing function for filtering the load data that are used as input to the models. This filtering aims to eliminate the effect of unpredictable events (power cuts, black outs or communication faults with SCADA) from the input. As a consequence, the quality of the output forecasts is expected to improve.

Several methods are being developed for the LF task:

\section{Simple forecasting}

A simple algorithm will be implemented using the load value from the same time the previous week, scaled by a factor representing the change in load between the two weeks.

\section{Fuzzy neural network model}

This model receives past load values to produce forecasts for the next 48 hours. The model parameters are adapted as new data arrive. Special attention is paid for predicting the load of weekends and special days. Temperature forecast is not considered as input to the model since such data is not available. However, the model compensates on this factor through auto-adaptation. The model produces hourly forecasts.

\section{ARMA model}

This model is an autoregressive model.

All the above methods are to be integrated in the LFmodule. However, when the software runs on-line only one method will provide forecasts to the rest of the modules (UC, ED) of the MORE-CARE software.

\section{WIND POWER FORECASTING}

The purpose of the Wind Power Forecasting (WPF) module is to provide forecasts for the power output of each wind farm connected to the power system, as well as the corresponding confidence intervals. Forecasts are required by the ED and UC functions of MORE CARE software. More specifically, models need to be developed to forecast:

- Average wind power output of a wind park. The average power is preferable to the total power since it is useful for the planning functions. Models that predict directly wind power are based on SCADA data and time-series analysis. Forecasts are accurate for limited horizons of a few hours (i.e. up to 8-10 hours).

- $\quad$ Alternatively, wind speed can be forecasted and then converted to power using the wind turbine characteristic curve (power output vs. wind speed) or other filters. Wind speed forecasting models can be based on timeseries analysis for the short-term (up to 8-10 hours) or meteorological information for the long term (48 hours).

A number of different methods (e.g. persistence, ARMA, fuzzy models, meteorological models etc.) will be integrated in the module to perform the wind forecasting function. Time-series models will be activated to provide forecasts for the short-term (e.g. up to 4 hours ahead), while meteorological models will provide forecasts for the long term (e.g. up to 48 hours).

Array effects (which are likely to be direction-dependent) may cause the total wind power generated by a wind farm to be less than might be expected from simply applying the average load curve to the estimated wind speed and multiplying by the number of turbines. Therefore, provision will be made to enter an array effect index to scale the forecast power output of a given wind farm.

The WPF module will be developed in a generic way so as to be able to handle future installations of wind farms in the power system.

Finally, the user will be able to compare historical predictions with actual output data where available to enable tracking of the performance of the wind forecasting tool.

The following methods are being developed:

\section{Simple Forecasting}

Persistence consists on using the most recent value of wind speed or power as forecast for the entire planning horizon. Instead of using Persistence, one can use averages of past values as predictors (Naive predictors). Off line analysis permits to define the outperforming simple method for each case study.

\section{Linear ARMA models}

\section{Fuzzy-neural models}

Fuzzy autoregressive models are applied to predict wind speed or power up to 8-10 hours ahead. The input is past wind speed or power values as well as exogenous input (i.e. wind direction). The model parameters are self-adapted as new data arrive. Several tests using wind speed or power time series have shown that fuzzy logic based models outperform persistence.

\section{Meteorological information based model A}

A long-term (up to 48 hours) wind power forecasting module is also being developed. The module will be as adaptable as possible to varying availability of input data. To achieve the long term forecast, data input from a meteorological forecast model, such as HIRLAM, is required for one or more wind farms in the system. To make a shorter term, statistical correction, on-line SCADA data is required for one or more wind farms in the system. It is anticipated that the accuracy of the wind power forecast will increase with the proportion of wind farms having specific meteorological forecasts and/or on-line measurements. Where no time series input data is available for a wind farm, multipliers will be used to estimate the output from neighboring locations. This feature may also be useful in the event of temporary communications problems.

\section{Meteorological information based model B}

This module makes site specific wind speed forecasts based on the information produced by the SKIRON system of the Atmospheric Modeling \& Weather Forecasting Group of the University of Athens. The SKIRON meteorological forecasts cover a grid of $15 \times 15 \mathrm{~km}$ of Crete.

The operator will have the possibility through the MMI, to choose any of the above methods for on-line use. Special attention is paid to combine the short-term and long-term wind forecasts provided by the statistical and meteorological models respectively.

\section{UNIT COMMITMENT}

The objective of the Unit Commitment (UC) function is to determine the combination of production sources and units that will supply the expected demand of energy over the future period in question. This combination is the result 
of an optimization procedure that takes into account economic criteria and is subject to different types of economical, technical and security constraints.

The UC module of the MORE CARE software will suggest to the operator of the power system a secure operating scenario for the next hours. In an interconnected power system, Unit Commitment is performed usually once a day or more, and is based solely on load forecasts. In isolated systems with high penetration from renewable (i.e. $>20 \%$ ), it is necessary to update regularly the proposed Unit Commitment schedules in order to account for the variability in the production of the renewable. Thus, the UC models developed operate in a "sliding window" scheme.

The length of the look-ahead time (planning horizon) depends on the type of the installed power units. Depending on the type of units that participate in the UC process, two UC functions are defined:

- 48 hour ahead UC: This is essential when steam units exist in the power system, so the horizon needs to cover a long period up to one or two days. Hourly time-steps are considered. This function is performed every hour and it includes a pre-dispatch calculation. The output of this module is checked against the security structures. If an insecure output is detected, a new UC is required.

- 4 hour ahead UC: This function is performed for a shorter horizon of 4 hours. The operation plan of the steam units is considered as known by the 48 hour UC function. The objective is to optimize the scheduling of the rest of units using a smaller time-step of a few minutes, e.g. 20minutes taking into account the updated load and renewable forecasts.

On operator's request, the operating restrictions will include preventive control measures on the UC module, coming from the security assessment module.

Two models are developed within the MORE CARE project for the UC task. Both models are based on Genetic Algorithms (GA) and are able to simulate systems with steam units, gas turbines, diesel and combined cycle power units including renewable like wind farms and small hydro stations. The operator will have the possibility to select one of them for on-line use.

\section{Genetic algorithm A}

This model is based on a Genetic Algorithm optimization technique. It is suitable for both the 48 hour ahead and the 4 hour ahead UC functions:

- Firstly, the 48 hour ahead UC function is performed in order to provide an hour-by-hour schedule of the power units operation for the next horizon.

- The output of the 48 hour ahead UC function is used as input (in the form of soft constraints) to the 4 hour ahead UC function in order to provide a schedule for all the units for the next 4 hours every 20 minutes.

The new models comprise substantial extensions of previous Unit Commitment models, namely by including hydro power with pumping and market issues.

Regarding hydro power, a simplified model for the hydro system was developed, where cascading, pumping and other dependencies between reservoirs are included. Decisions about generation and pumping are subject to reservoir con- straints, taking into account affluences (that come from the forecasting modules), reservoir limits and end-of-period goals. These models are included in the fitness function calculation, leading to optimal hydro-thermal-wind power integrated management.

Consideration of contracts and market issues (both in UC and ED) lead to the consideration of the following cases:

- Bilateral contracts (assumed feasible), by considering $\Delta \mathrm{P}$ both in generator (if included) and in load.

- Contractual obligations for producers, namely limit in power (min P) in some intervals, scheduled units, number of hours of operation (wind generation).

More complex situations are not likely to occur in islands, due to their special characteristics, and their exception to the liberalized electricity markets.

\section{Genetic algorithm $B$}

The other GA based module follows a different approach. It has a first step that preprocesses the demand curve, by subtracting the wind power forecast per hour to the demand of that hour. Bilateral contracts are also treated as differences on the load curve.

Then, a special routine is applied to determine the operating status of the hydroelectric units within a range of $24 / 48 \mathrm{~h}$. This routine is based on linear programming and determines the power production or consumption for each hydroelectric plant so as to minimize the energy production costs (based on the load curve) while satisfying the reservoir constraints, the inflows/outflows constraints, as well as the target volume at the end of each UC cycle.

For the thermal units commitment an integer valued $\mathrm{Ge}$ netic Algorithm (GA) is developed. This choice is made in an effort to reduce the chromosome size and increase the efficiency of the algorithm. Instead of creating a big binary chromosome that consists of the ON/OFF unit hourly-states during the Unit Commitment (UC) cycle, the integer GA chromosome consists of the ON/OFF unit cycles during the Unit Commitment cycle. Special operators are also implemented to assist and accelerate the algorithms.

\section{ECONOMIC DISPATCH}

The objective of the Economic Dispatch (ED) function is to distribute the load among the generator units selected in the Unit Commitment so that the total cost of the power system operation is minimized. The ED function is performed only for the basic time-step (e.g. 20 minutes ahead) of the planning horizon in order to provide the generators set-points to the operators.

The ED module is possible to compute the optimal generation production satisfying constraints of circuit loads, bus voltages and reactive generation to satisfy the load demand and losses of the system. It can also consider constraints related to bilateral contracts and independent producers of renewable power sources according to specific terms and conditions. On operator's request the output of the Economic Dispatch module (set-points) can be checked against security constraints with the security monitoring module, but these constraints will not be fed back as input to the ED modules.

Two different approaches are developed in MORE CARE 
to perform the economic dispatch function. The operator will have the possibility to select the preferred approach as a configuration parameter of the advice system.

\section{Optimal Power Flow based on Linear Programming}

This module uses a linear model of the power system, which relates the generation production rescheduling and the other control resetting to the operating cost and the transmission network constraints. For the operation of a conventional unit the operating cost is specified by a cost curve expressing either running cost, dependent on unit's characteristics, or selling price (bidding) in case of private owners. For the Wind Parks discrete values can be achieved by switching on or off a number of wind turbines. Pump storage hydro units may operate either producing energy injected to the power system energy using the water stored, or consuming energy to pump water into the reservoir. Loads may operate under contractual agreements, between the consumer and an independent power producer, and /or between the consumer and the utility, defining maximum and minimum requested power.

\section{Genetic algorithms technique}

The Economic Dispatch module based on GA schedules the operating points of the network's thermal generators for the next hour. The module uses a real-coded genetic algorithm to minimize the operating cost while satisfying operating limits (ramp rates included), power balance and network constraints (optional).

Besides decreasing the execution time of the previous version, the new algorithm implements two main additions to the CARE module.

- Inclusion of network restrictions in the form of line power flow limits. The DC optimal power flow that is performed insures that the solution produced is viable. The execution time for this is still relatively small (a few seconds) comparing to the CARE ED module.

- The module is capable of handling non-convex cost functions (valve points, etc).

\section{ON-LINE DYNAMIC SECURITY}

In autonomous power systems, dynamic security assessment is a key issue in the operation and management of the networks. In fact, sudden changes of system operating conditions must be quickly and efficiently compensated by generators to avoid frequency excursions or high $\mathrm{df} / \mathrm{dt}$ variations, which may trigger the operation of system frequency relays, like under frequency protection relays of generators, provoking system collapse. This means that expected system frequency excursions and $\mathrm{df} / \mathrm{dt}$ values, for some disturbances, must be assessed in a fast way to help in defining the more robust operating strategies. In addition, under- or overvoltages might disconnect generation and have to be guarded against.

On-line dynamic analysis of system behavior for a number of pre-specified disturbances is practically impossible using conventional tools. "Learning from examples" techniques, e.g. Decision Trees (DT) or Artificial Neural Networks (ANN), are used to provide accurate and fast evaluation of system stability by defining security rules and secu- rity functions. These structures need to be extracted from a "Learning Set" and can be used for on-line monitoring of the appropriately defined security margin and as security restrictions in the UC and ED modules, in order to arrive at the most economic and secure operating strategies.

\section{Off-line Procedure for the Model Development}

A learning set (LS) is therefore required in order to extract from it the knowledge needed to derive security structures. It consists of a large number of operating points (OPs), covering the most probable operating scenarios and strategies of the power system under study, in order to ensure its representativity. Each OP is characterized by a vector of pre-disturbance steady-state variables, called attributes, which can be either directly measured (power, voltages etc.) or indirectly calculated quantities (wind penetration, spinning reserve etc.). The quality of the selected attributes and the representativity of the LS affect critically the performance of the automatic classification structures.

It should be stressed that in order to provide corrective actions in the MORE CARE software the LS needs to be created, for each selected disturbance. For Crete, LS for dynamic security assessment (DSA) were created, considering as attributes only frequency related variables, while for dynamic security monitoring (DSM), the LS were obtained including also voltage variations variables, as in this network voltage problems are sometimes an issue of concern. Thus the DSA LS should mainly emulate the unit dispatching policy, while the DSM LS should represent a more complete picture of the system including voltage related variations. As a result, a larger and more complex LS should be created for DSM in this case.

For the Madeira network three LS were created, one for each selected disturbance, to derive the security structures for both the DSA and DSM functions. In the Madeira system the main concern is frequency. More specifically, the security indices that characterize the safety of this system are the minimum value reached by frequency deviation and the maximum value of $\mathrm{df} / \mathrm{dt}$ regarding only negative rates.

The performance and the reliability of the designed classifiers is evaluated with an independent test set, which has the same structure as the LS, i.e. it is generated in exactly the same way. In this way the capability of the developed structures to classify correctly unforeseen states can be evaluated on a relatively objective basis.

Due to the importance of the dynamic behavior in the power system operation, the MORE CARE system includes two modules responsible for the evaluation of dynamic security relatively to a number of pre-specified disturbances:

\section{The Dynamic Security Assessment Module (DSA)}

The dynamic security assessment module has the task of evaluating if a given unit commitment dispatch policy is dynamically secure. This evaluation is performed exploiting "learning from example" techniques. However, unlike the CARE system, in case an insecure situation is detected, a new UC solution is obtained including relevant security constraints, through the introduction of penalties in the fitness function of the GA approach used to obtain the UC solution. The security restrictions can be obtained from one of the two following procedures: 
- provided in the form of rules extracted from the DT;

- provided by the output of the ANN.

In this case the GA approach will provide very fast solutions, since only a few generations will be needed to get the new UC solution, as the first population is made of the insecure solution obtained initially.

Another available approach will be only to exploit backward the DT, from the insecure leaf where the first UC solution arrived, and find out a new secure UC solution. However in this case there is no optimality concern in determining the UC solution.

\section{The Dynamic Security Monitoring Module (DSM)}

The dynamic security monitoring module is used, upon operator request, for evaluating the dynamic robustness of the economic dispatch module and the present system operating state. As in the DSA module, the DSM will also provide corrective control measures, i.e. the produced generator set points and voltages will be modified, in case an insecure state is detected. Unlike the DSA module however, the corrective security rules will not be fed back to the ED module. When insecurity is detected for the disturbances considered as critical by the operator, active power redispatch can be performed exploiting rules derived from the DTs or through sensitivity of neural networks. Note, however, that this redispatch can, in some cases, be an insufficient preventive control measure because no solution is available. In addition, these control measures will now concern not only frequency, but also voltage deviations of the system, like in the case of Crete.

Two main approaches are developed for the fast security assessment task:

\section{Decision Trees Method}

The Decision Trees method, uses an inference inductive procedure, and derives classification structures of the type "if-then-else", able to provide a fast secure or insecure classification of each operating point. The DTs provide an overview and understanding of the dependence of the system's security on its pre-disturbance state. They are suitable for corrective control purposes. Thus, if the proper control variables are used as candidate attributes, they are able to provide explicit and quantitative information about the actions to be taken, if a potentially unsafe operating state is detected. The DTs are readily converted to a set of rules that can be very easily stored and incorporated in the security assessment software.

\section{Artificial Neural Networks Method}

The Artificial Neural Networks (multi-layer perceptron type), uses as inputs relevant system attributes to provide as output an emulation of the system robustness degree for the disturbances under analysis, i.e. the frequency deviation and the derivative of frequency relatively to time (df/dt). A previous feature selection stage is performed to select from the initial set of characterizing features the most relevant ones that are less correlated among each other. A new approach is used here for this purpose, consisting in exploiting sensitivities of the outputs of an initial ANN, relatively to the input variables.

\section{CONCLUSIONS}

This paper presents the main features of MORE CARE, an advanced control system that aims to provide advice to power system operators for the optimal operation and management of isolated power systems with large integration from renewable power sources. A number of different models for performing each main task have been integrated, so the operator can choose which models will run on-line.

\section{REFERENCES}

[1] ARMINES, NTUA, INESC, RAL, PPC Development and implementation of an advanced control system for the optimal operation and management of medium-sized power systems with a large penetration from renewable power sources, Final report of JOULE II project JOU2-CT92-0053. Edited by the Office for Official Publications of the European Communities, Luxembourg 1996.

[2] NTUA, INESC, ARMINES, RAL, PPC, Final Report of JOR3-CT960119, "Advanced Control Advice for Power Systems with Large Scale Integration of Renewable Energy Sources", September 1999.

[3] Wind Engineering, Vol. 23, No. 2, 1999, CARE Special Issue, 